\title{
Логический плюрализм и неклассическая теория категорий
}

\author{
В. Л. ВАСЮКОВ
}

\begin{abstract}
Logical pluralism prove to be much more intruiging phenomenon if we envisage its impact on elementary logical theories. Breaking the tenet of the unique (namely, classical) logical basis for those we find ourself in the realm of non-classical elementary logical theories based on the various non-classical logics. It is expecially important if we take into account that such theories underlie non-classical mathematics according to the apt slogan "there are as many mathematics as logics" - suffish it to recall relevant arithmetic, quantum set theory, fuzzy set theory, paraconsistent mathematics etc. In the paper non-classical axiomatic category theories are approached which are based on some non-classical categorical constructions.
\end{abstract}

Keywords: pluralism, non-classical categories, axiomatic category theory, category theory of categories

\section{1 Введение}

Сегодня логический плюрализм представляется совершенно естественной точкой зрения для большинства работающих логиков и кажется тривиальным обсуждать влияние, которое он оказывает на логические исследования в целом. Мы знаем, что существует множество разнообразных логических систем, а их взаимовлияние и соперничество представляется достаточно интересной сферой исследования современной логики и методологии (см. [6]). Но ситуация оказывается гораздо более интригующей, если мы обратимся к рассмотрению следствий логического плюрализма для элементарных логических теорий, где молчаливо предполагается, что в их основании лежит классическая логика.

Мы по-разному оцениваем эти последствия, принимая во внимание особенности семантики неклассических логических систем. Это вызвано тем, что мы можем интерпретировать элементарные теории в 
- структурах, основанных на классической логике, и

- структурах, основанных на некласических логиках.

Дело в том, что при рассмотрении теоретико-множественных конструкций мы, как правило, подразумеваем, что с формальной точки зрения абстрактная теория множеств является элементарной логической теорией, основывающейся на классической логике. И как таковая она может быть модифицирована в своей логической части. Например, у нас имеется нечеткая теория множеств $F Z F$, основанная на нечеткой логике $F L$ [13], квантовая теория множеств на базе квантовой логики [12], паранепроттиворечивая теория множеств $Z F_{1}$, основанная на паранепротиворечивой логике да Косты [8], и т.д. Мы вольны выбрать любую из них для конструирования требуемых нам моделей (и множеств, которые нам нужны).

Но это не единственный способ. Мы можем использовать логические теории, основанные на классической логике (которые обычно рассматриваются без всякого обоснования как единственные настоящие элементарные логические теории), интерпретируя в них неклассические теории или эксплуатируя с этой целью конструкции соответствующих фрагментов классических элементарных теорий (даже не осознавая этого).

Следует также принять во внимание, что теории множеств не являются единственными подобными теориями. Нетрудно указать в связи с этим, например, на конструкцию релевантной арифметики [10], которая разработана как раз согласно такому «плюралистическому» алгоритму на базе релевантной логики. Другой пример еще более показателен - К.Мортенсен описывает противоречивую математику на базе паранепротиворечивой логики [11]. Похоже, что к данной ситуации более всего подходит лозунг, годящийся для всех этих случаев - «существует столько математик, сколько и логик».

Попробуем прояснить подробности этого лозунга на примере теории категорий, которая может быть преобразована в интересный пример неклассической элементарной логической теории.

\section{2 Встраивание неклассических логик}

Мы обнаруживаем множество неклассических логик в моделях, основанных на классических логиках. Среди них семантика воз- 
можных миров, алгебраическая семантика, топологическая семантика - просто потому, что они используют конструкции, построенные из множеств, источником которых является абстрактная теория множеств.

В теории категорий мы получаем интерпретации неклассических логик путем использования топосов функторов из малых категорий в категорию множеств Set. В качестве же множествобъектов категории $\mathrm{Set}$ - мы берем классические множества абстрактной теории множеств Цермело-Френкеля $Z F$ и сопоставляем их элементам алгебраической модели соответствующей неклассической логики. Именно так мы получаем интерпретацию интуиционистской логики путем использования алгебры Гейтинга $P$ в роли малой категории и строя оценку интуиционистских формул с помощью $\operatorname{Set}^{P}$-оценки $V: \Phi_{0} \rightarrow \operatorname{Set}^{P}(1, \Omega)$, которая выбирает некоторое множество для любого элемента $P$ (см. [4]).

Если мы, распространяя этот метод на другие логики, попытаемся заменить алгебру Гейтинга на другую, например, алгебру да Косты, то мы сталкиваемся с проблемой интерпретации отрицания: в алгебре Гейтинга отрицание не является примитивной операцией в отличие от алгебры да Косты, потому совершенно неясно, как действовать в этом случае. Выход заключается, по-видимому, в использовании конструкции так называемых $N$ категорий. В рассматриваемом случае нам нужна $C N$-категория $C$, которая является категорией предпорядка, снабженной контравариантным функтором $N: \mathcal{C} \rightarrow \mathcal{C}$, таким, что

(i) в $\mathcal{C}$ имеются конечные произведения $\langle-,-\rangle$, копроизведения $[-,-]$ и $\mathcal{C}$ является дистрибутивной решеткой по отношению к ним, т.е.

$\langle[a, b],[a, c]\rangle \cong[a,\langle b, c\rangle]$ имеет место для любых объектов $a, b, c$ из $\mathcal{C}$;

(ii) в $\mathcal{C}$ имеется инициальный объект 0 и терминальный объект $1,1 \cong[a, N a]$ и $0 \cong\left\langle a^{0}, N a^{0}\right\rangle$, где $a^{0}=N\langle a, N a\rangle$;

(iii) для любого объекта $a$ из $\mathcal{C}$ имеются стрелки $N^{2} a \rightarrow a$ и $a^{0} \rightarrow(N a)^{0}$ в $\mathcal{C}$

(iv) $\mathcal{C}$ допускает экспоненцирование; 
$(\mathbf{v}) a \rightarrow b$ является стрелкой в $\mathcal{C}$ тогда и только тогда, когда $a \Rightarrow b \cong 1$ для любой пары объектов $a, b$ в $\mathcal{C}$, где $a \Rightarrow b$ есть экспоненциал;

(vi) для любой пары объектов $a, b$ в $\mathcal{C}$ имеется стрелка $a^{0} \rightarrow$ $(b \Rightarrow a) \Rightarrow((b \Rightarrow N a) \Rightarrow N b)$ в $\mathcal{C}$.

Теперь, строя топос $S e t^{\mathcal{C}}$, мы получаем интерпретацию формул паранепротиворечивой логики да Косты $C_{1}$,которая будет основана на $S e t^{\mathcal{C}}$-оценке, представляющей собой функцию $V$ : $\Phi_{0} \rightarrow \operatorname{Set}^{\mathcal{C}}(1, \Omega)$, сопоставляющую каждой пропозициональной переменной $\pi_{i}$ некоторое истинностное значение $V\left(\pi_{i}\right)=1 \longmapsto \Omega$ (см. [14]).

Другим примером является интерпретация релевантной логики $R$ в категории $S e t^{\mathcal{A}}$-функторов из $R N$-категории $\mathcal{A}$ в категорию множеств $\operatorname{Set}[1]$. Здесь $R N$-категория $\mathcal{A}$ является (групоидальной) категорией предпорядка, наделенной ковариантным бифунктором $\otimes: \mathcal{A} \times \mathcal{A} \rightarrow \mathcal{A}$, таким, что

(i) в $\mathcal{A}$ имеются конечные произведения $\langle-,-\rangle$, копроизведения $[-,-]$ и $\mathcal{A}$ дистрибутивна по отношению к ним;

(ii) для любых объектов $a, b, c$ в $\mathcal{A}$ имеются следующие естественные изоморфизмы:

$a \otimes[b, c] \cong[a \otimes b, a \otimes c]$,

$[b, c] \otimes a \cong[b \otimes a, c \otimes a]$,

т.е. бифунктор сохраняет копроизведения;

(iii) $\mathcal{C}$ допускает экспоненцирование относительно $\otimes$, т.е. следующая диаграмма коммутирует

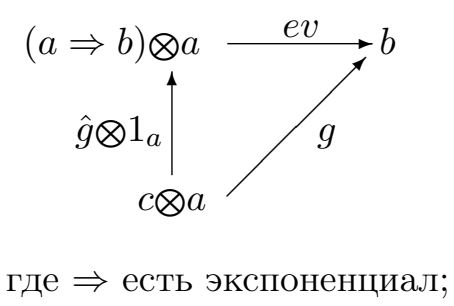

(iv) выполняются следующие функторные уравнения: 
(a) $\left(g_{1} f_{1}\right) \otimes\left(g_{2} f_{2}\right)=\left(g_{1} \otimes g_{2}\right)\left(f_{1} \otimes f_{2}\right)$;

(b) $1_{A} \otimes 1_{B}=1_{A \otimes B}$.

Интерпретация формул $R$ здесь основывается на $S e t^{\mathcal{A}}$-оценке, являющейся функцией $V: \Phi_{0} \rightarrow \operatorname{Set}^{\mathcal{A}}(1, \Omega)$, сопоставляющей каждой пропозициональной переменной $\pi_{i}$ некоторое истинностное значение $V\left(\pi_{i}\right)=1 \longmapsto \Omega$.

Еще одним случаем является интерпретация квантовой логики в топосе $S e t^{\mathcal{E}}$, где $\mathcal{E}$ есть ортомодулярная решетка, которая может рассматриваться в некотором смысле как перевод квантовой логики (основанной на ортомодулярной решетке) в контекст универсума множеств [2]. Здесь $\mathcal{E}$ есть ортомодулярная категория, т.е. категория, снабженная контравариантным функтором $N: \mathcal{E} \rightarrow \mathcal{E}$, таким, что

(i) в $\mathcal{E}$ имеется инициальный объект 0 и терминальный объект 1

(ii) в $\mathcal{E}$ имеются конечные произведения $\langle-,-\rangle$ и конечные копроизведения [-.-];

(iii) $N^{2} a \cong a$ для любого объекта $a$ в $\mathcal{E} ;\langle a, N a\rangle \cong 0,[a, N a] \cong 1$ для любого объекта $a$ в $\mathcal{E}$;

(iv) $N[a, b] \cong\langle N a, N b\rangle, N\langle a, b\rangle \cong[N a, N b]$ для любой пары объектов $a, b$ в $\mathcal{E}$.

(v) если $a \rightarrow b$ есть стрелка в $\mathcal{E}$, то $[a,\langle N a, b\rangle] \cong b$ для любых двух объектов $a, b$ в $\mathcal{E}$.

Соответствующая интерпретация некоторых систем квантовой логики получается таким же образом, путем использования $S e t^{\mathcal{E}}$-оценки.

\section{3 Неклассические категории}

По-видимому, топосы являются не единственными категорными универсумами, пригодными для интерпретации неклассических логик. Более тщательный анализ этого вопроса показыает, что мы можем расширить конструкцию топоса подобно тому, как 
вводятся неклассические теории множеств, для получения более естественной категорной семантики неклассических логик. Первым шагом на этом пути будет рассмотрение конструкции nотоса или monoca да Koсты - категорного эквивалента алгебры да Косты (см. [3]).

Потос представляет собой паранепротиворечивый универсум, в котором можно развивать паранепротиворечивую математику подобно тому, как это было сделано в случае интуиционистской математики в топосах. В потосе паранепротиворечивость абсолютно имманентна и, более того, она лежит в основе всех конструкций, она глобальна и универсальна. Здесь уже классическая математика выступает как артефакт в паранепротиворечивом универсуме, как некоторое локальное отклонение от паранепротиворечивой регулярности. Таким образом, например, при интерпретации $C_{n}$-систем можно использовать неистинностнофункциональную оценку, в то время как истинностно-функциональная оценка становится характеристикой лишь случая булевых топосов, которые теперь являются частным случаем потосов.

Более формально, потос $C$ можно определить как декартово замкнутую категорию, которая также комплементарно замкнута и содержит классификатор подобъектов. Комплементарная замкнутость $C$ означает, что

(i) для любого объекта $a$ из $C$ имеется объект $a^{\prime}$, такой, что в $C$ существуют стрелки $a^{\prime \prime} \rightarrow a$ и $a^{o} \rightarrow\left(a^{\prime}\right)^{o}$, где $a^{o}=\left\langle a, a^{\prime}\right\rangle^{\prime}$,

(ii) $1 \cong\left[a, a^{\prime}\right], 0 \cong\left\langle a^{o}, a^{o \prime}\right\rangle$,

(iii) для любых двух объектов в $C$ имеется стрелка $a^{o} \rightarrow(b \Rightarrow$ $a) \Rightarrow\left(\left(b \Rightarrow a^{\prime}\right) \Rightarrow b^{\prime}\right)$, где $b \Rightarrow a$ представляет собой экспоненциал.

Как следствие введенной новой конструкции приходится рассматривать новую категорию паранепротиворечивых множеств $P S e t$, где в качестве объектов используются $Z F_{1}$-множества паранепротиворечивой теории множеств (см. [8]). Система $Z F_{1}$ согласуется с теорией множеств Цермело-Френкеля $Z F_{0}$ так же, как паранепротиворечивое первопорядковое исчисление с равенством согласуется с классическим. В то же время категория 
$P$ Set оказывается не топосом, но потосом множеств, и поэтому может быть построена интерпретация в потосе $\operatorname{PSet}^{A}$ и можно доказать полноту $C_{1}$ по отношению к подобной семантике.

Рассмотренная выше конструкция топоса $S e t^{\mathcal{E}}$, где $\mathcal{E}$ является ортомодулярной решеткой, может рассматриваться, в некотором смысле, как перевод квантовой логики (основанной на ортомодулярной решетке) в контекст интуиционистского универсума, поскольку Set представляет собой топос (будучи интуиционистской конструкцией по своему происхождению). Но всегда ли нам нужен именно интуиционистский универсум в качестве основы подобного рассмотрения? Представляется более стественным использовать категории, содержащие квантовую логику в качестве своей внутренней структуры. Существуют формулировки квантовой теории множеств (см. [12]) $Z F$, которые отличаются от классической теории множеств, и это позволяет думать, что подобные множества не порождают топос. В этом случае $Q S e t^{\mathcal{E}}$ (где QSet является категорией квантовых множеств) не будет топосом и рассмотренная выше интерпретация проваливается.

Чтобы обойти подобные трудности, можно рассмотреть конструкцию квантоса, которую можно охарактеризовать как неклассическую модификацию топоса с некоторой дополнительной структурой, позволяющей истолковать частичность отрицания в квантовой логике [15]. Квантос $\mathcal{Q}$ представляет собой биполную категорию, которая также комплементарно замкнута, ортомодулярна и имеет классификатор подобъектов $\Omega$. Комплементарная замкнутость означает, что

(i) для любого объекта $b$ из $\mathcal{Q}$ имеется объект $a^{\prime}$, такой, что $a^{\prime \prime} \cong a$ и для любой стрелки $a \rightarrow b$ в $\mathcal{Q}$ имеется стрелка $b^{\prime} \rightarrow a^{\prime}$

(ii) $\left\langle a, a^{\prime}\right\rangle \cong 0,\left[a, a^{\prime}\right] \cong 1$ для любого объекта $a$ из $\mathcal{Q}$;

(iii) $[a, b]^{\prime} \cong\left\langle a^{\prime}, b^{\prime}\right\rangle,\langle a, b\rangle^{\prime} \cong\left[a^{\prime}, b^{\prime}\right]$ для любых двух объектов $a, b$ из $\mathcal{Q}$.

Ортомодулярность означает, что

(iv) если $a \rightarrow b$ есть стрелка в $\mathcal{Q}$, то $\left[a,\left\langle a^{\prime}, b\right\rangle\right] \cong b$ для любых двух объектов $a, b$ из $\mathcal{Q}$. 
Категория QSet оказывается квантосом и точно так же доказывается, что категория $Q S e t^{\mathcal{E}}$ тоже будет квантосом. Одни и те же системы квантовой логики интерпретируются в обоих типах квантосов гораздо более естественно, чем это делается в случае $S e t^{\mathcal{E}}$. В некотором смысле квантосы можно рассматривать как квантовый категорный универсум для квантовологических исследований.

\section{4 Классическая элементарная теория категорий}

Наконец, как уже было сказано, с логической точки зрения теория категорий может рассматриваться как элементарная теория, чьи «категорные» нелогические аксиомы добавляются к первопорядковому исчислению с равенством. Подобный подход был реализован в 60-70-х гг. У. Хэтчером, Ж. Бланом и М.-Р. Доннадью и другими. Формально подход Блана и Доннадью можно описать следующим образом.

Язык элементарной аксиоматизированной теории категорий $E T A C$ определяется с помощью

(i) счетного множества переменных двух типов: переменных типа объект $x_{1}, x_{2}, \ldots$ и переменных типа стрелки $f, g, h, \ldots$;

(ii) логических констант: $\Rightarrow, \Leftrightarrow, \wedge, \vee, \neg, \exists, \forall,=$;

(iii) тернарного предиката $D(-,-,-)$, где первая переменная есть переменная типа стрелки, а две остальные являются переменными типа объект $\left(D\left(f, x_{1}, x_{2}\right)\right.$ означает « $f$ есть стрелка из $x_{1}$ в $\left.x_{2} \gg\right)$;

(iv) тернарного предиката $\Gamma(-,-,-)$, где все переменные являются переменными типа стрелки $(\Gamma(f, g, h)$ означает $\ll h$ есть композиция $f$ и $g \gg)$.

$E T A C$ аксиоматизируется с помощью следующих схем аксиом:

Ax1. $\forall f \exists ! x_{1}, x_{2}\left[D\left(f, x_{1}, x_{2}\right)\right]$

Ax2. $\forall x_{1} \exists i\left[\Phi\left(x_{1}, i\right) \wedge D\left(i, x_{1}, x_{1}\right)\right]$, где $\Phi\left(x_{1}, i\right)$ есть формула

$$
\forall f, g, x_{2}, x_{3}\left[D\left(f, x_{1}, x_{2}\right) \wedge D\left(g, x_{3}, x_{1}\right) \Rightarrow \Gamma(i, f, f) \wedge \Gamma(g, i, g)\right]
$$


Ax3. $\forall h \Gamma(f, g, h) \Rightarrow \exists x_{1}, x_{2}, x_{3}\left[D\left(f, x_{1}, x_{2}\right) \wedge D\left(g, x_{2}, x_{3}\right) \wedge\right.$ $\left.D\left(h, x_{1}, x_{3}\right)\right]$

Ax4. $D\left(f, x_{1}, x_{2}\right) \wedge D\left(g, x_{2}, x_{3}\right) \Rightarrow \exists h \Gamma(f, g, h)$

Ax5. $\Gamma(f, g, h) \wedge \Gamma(f, g, h \prime) \Rightarrow h=h^{\prime}$

Ax6. $\Gamma(f, g, k) \wedge \Gamma(g, h, l) \wedge \Gamma(f, l, m) \wedge \Gamma\left(k, h, m^{\prime}\right) \Rightarrow m=m^{\prime}$

Интуитивный смысл этих аксиом можно было бы передать с помощью следующих диаграмм:

Ax1. $x_{1} \stackrel{f}{\rightarrow} x_{2}$

Ax2.

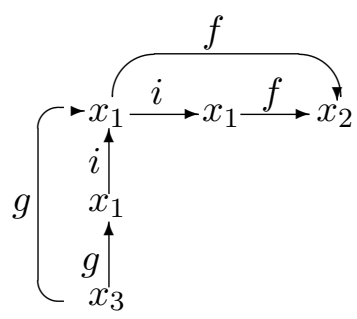

$\mathrm{Ax} 3, \mathrm{Ax} 4, \mathrm{Ax} 5$.

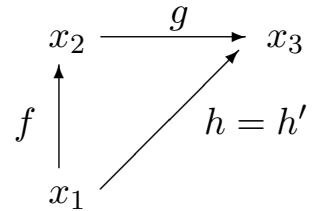

Ax6.

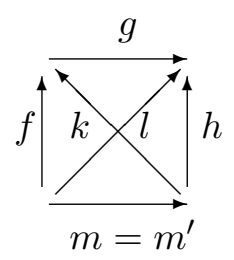

Для описания структуры категорий нам необходим метаязык, позволяющий описывать строение конкретной категории. Подобный язык элементарной теории категории категорий $C C$ образуется путем добавления к языку $E T A C$ следующих нелогических символов: 
- символы констант типа объект 0,1,2,3;

- символы констант типа стрелка $\sigma, \delta_{0}, \delta_{1}, \tau, \alpha, \beta, \gamma$;

- | - |, функциональный символ, определенный на переменных типа объект с областью значений на переменных типа стрелка;

- $d_{(-)}$, функциональный символ, определенный на переменных типа объект с областью значений на переменных типа стрелка.

Аксиомы $C C$ выглядят следующим образом:

Ax2-1. Конъюнкция всех аксиом $E T A C$.

Ax2-2.

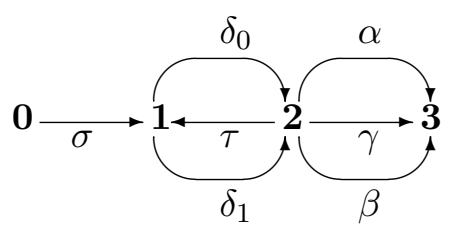

где все константы различны, 0 является инициальным объектом, 1 является терминальным объектом и $\mathbf{2}$ есть генератор.

ОПРЕДЕЛЕНИЕ 2-1. Интерпретация формуль ЕТАС в терминах объектов $C C$. Пусть ф будет регулярной формулой в языке $E T A C$ (т.е. такой, что в ней нет свободных переменных, каждая переменная связана квантором). Известно, что всякая формула является эквивалентной регулярной формуле. Пусть $A, B$, $\ldots, C$ есть $n$ переменных типа объект со свободными переменными типа объект из $\phi$, и пусть $f, g, \ldots, h$ будут $m$ переменными типа стрелка со свободными переменными типа стрелка. Пусть $a, b, \ldots, c ; x, y, \ldots, z$ будут $m+n$ переменными типа стрелка, отличные друг от друга и отличные от $f, g, \ldots, h$, и $A^{\prime}$ есть переменная типа объект. Определим формулу теории $C C$

$$
\Phi^{*}\left(A^{\prime} ; a, b, \ldots, c ; x, y, \ldots, z\right)
$$


которую для удобства назовем интерпретацией $\phi$ в $A^{\prime}$, индукцией по длине формулы. Если ф есть $D(f, B, C)$, тогда $\Phi^{*}(A ; x, b$, c) будет формулой

$$
x: 2 \rightarrow A \wedge b: 1 \rightarrow A \wedge c: 1 \rightarrow A \wedge \delta_{0} x=b \wedge \delta_{1} x=c .
$$

Если $\phi$ есть $(f, g, b)$, тогда $\Phi^{*}(A ; x, y, z)$ (обозначаемая так же как $\left.x \cdot{ }_{A} y=z\right)$ будет формулой

$$
\bigwedge_{u=x, y, z} u: 2 \rightarrow A \wedge \exists t: 3 \rightarrow A(\alpha t=x, \beta t=y, \gamma t=z) .
$$

Если $\phi$ есть $\psi_{1} \wedge \psi_{2}$, тогда $\Phi^{*}\left(A^{\prime} ; a, \ldots ; x, \ldots\right)$ будет формулой

$$
\psi_{1}^{*}\left(A^{\prime} ; a, \ldots ; x, \ldots\right) \wedge \psi_{2}^{*}\left(A^{\prime} ; a, \ldots ; x, \ldots\right) .
$$

Если $\phi$ есть $\exists B \psi(B, C, \ldots ; f, \ldots)$, тогда $\Phi^{*}(A ; ; c, \ldots ; x, \ldots)$ будет формулой

$$
\exists b: 1 \rightarrow A \psi *(A ; b, c, \ldots ; x, \ldots),
$$

где $b$ отлично от $\{c, \ldots ; x, \ldots\}$. Если ф есть $\exists f \psi$, тогда $\Phi^{*}(A ; b, \ldots$; $y, \ldots)$ будет формулой

$$
x: 2 \rightarrow A \psi^{*}(A ; b, \ldots ; x, y, \ldots),
$$

где $x$ отлично от $\{b, \ldots ; y, \ldots\}$. Если $\phi$ есть $\neg \psi$, тогда $\Phi^{*}(A ; a, \ldots ;$ $x, \ldots)$ будет формулой

$$
\neg \psi^{*}(A ; a, \ldots ; x, \ldots) .
$$

Обозначим $A \vDash \phi$ для $C C \vdash \Phi(A)$. Говорят, что $x$ является: $x \in A$,

стрелкой в $A$ для $(C C \vdash x: 2 \rightarrow A)$, обозначая это как объектом в $A$ для $(C C \vdash x: 1 \rightarrow A)$, обозначая это как $x \in A$, элементом $A$ для $x$ есть стрелка или объект в $A$.

Ax2-3. $\forall A(A \vDash E)$, где $E$ представляет собой конъюнкцию аксиом $E T A C$.

Каждый объект $C C$ будет называться категорией, а каждая стрелка будет называться функтором. 
Ax2-4 (конечные предель и копределы). Для каждой пары категорий существует произведение и копроизведение. Для каждой пары функторов с общей областью и кообластью существует уравнитель и коуравнитель.

Ax2-5 (обратный образ суммы). Пусть

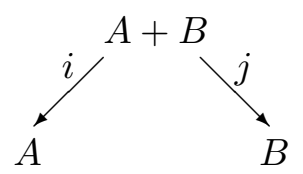

где $i$ и $j$ являются инъекциями суммы:

$(\forall x: 2 \rightarrow A+B)[(\exists y: 2 \rightarrow A(x=y i))$ или $(\exists y: 2 \rightarrow B(x=y j))]$.

Ax2-6. Это декартово замкнутая категория.

Обозначим $A^{B}$ экспоненциал $A$ и $B$ и

$$
e_{A}^{B}: B \times A^{B} \rightarrow A
$$

есть функтор оценки. $A^{B}$ и $e_{A}^{B}$ определяются с помощью свойства универсальности

$$
\forall f: B \times Z \rightarrow A \exists ! h: Z \rightarrow A^{B}\left[(1(B) \times h) e_{A}^{B}=f .\right.
$$

$h$ будет называться экспоненциально сопряженной с $f$ и будет обозначаться $\tilde{f}$.

ОПРЕДЕЛЕНИЕ 2-2 (дискретная категория). А является дискретной (дискретом) тогда и только тогда, когда

$$
\forall x: 2 \rightarrow A \exists y: 1 \rightarrow A(x=\tau y) .
$$

Ax2-7. $|A|$ является дискретом и $d_{A}$ является мономорфизмом и

$$
(\forall \text { дискрета } B)(\forall f: B \rightarrow A)(\exists ! h: B \rightarrow|A|)\left(h d_{A}=f\right) .
$$

ОПРЕДЕЛЕНИЕ 2-3 (символ $|-|$ для переменной типа стрел$\kappa u)$. Пусть $f: A \rightarrow B$, в таком случае $g=|f|$ тогда и только тогда, когда $d_{A} f=|f| d_{B}$. 
Ax2-8 (выбора). $(\forall f: A \rightarrow B)(A \supsetneqq 0$ и $B$-дискрет $\Rightarrow \exists g: B \rightarrow$ $A(f g f=f))$.

ОПРЕДЕЛЕНИЕ 2-4. Базис эскиза $\mathcal{E}$ состоит из трех категорий $\left(A_{1}, A_{2}, A_{3}\right)$ и функторов $d_{0}, d_{1}, c, e, a_{0}, a_{1}$ между этими категориями, таких, что:

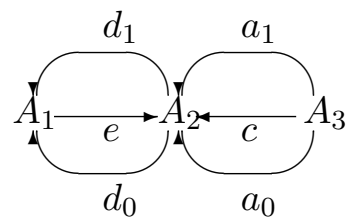

Базис эскиза $\mathcal{E}(A)$, ассочиированного с $A$, состоит из:

$$
\begin{gathered}
A_{i}=\left|A^{i}\right|(i=1,2,3) \quad d_{1}=\left|A^{\delta_{i}}\right|(i=0,1) \\
a_{0}=\left|A^{\alpha}\right| \text { и } a_{1}=\left|A^{\beta}\right| \text { и с }=\left|A^{\gamma}\right| .
\end{gathered}
$$

Нотация: $\mathcal{E}$ есть база эскиза,

$$
\Phi_{\mathcal{E}}\left(A_{4} ; b_{0}, b_{1}, e_{0}, e_{1}, f_{0}, f_{1}\right)
$$

обозначает соответствующую формулу:

$$
\begin{gathered}
A_{4} \underset{f_{i}}{\stackrel{b_{i}}{\rightrightarrows}} A_{5} \text { и } A_{2} \stackrel{e_{i}}{\rightarrow} A_{3}(i=0,1) \\
\text { и }\left\{\begin{array} { c } 
{ e _ { 0 } a _ { 1 } = d _ { 1 } c } \\
{ e _ { 0 } a _ { 0 } = I ( A _ { 2 } ) }
\end{array} \text { и } \left\{\begin{array}{c}
e_{1} a_{0}=d_{0} e \\
e_{1} a_{1}=I\left(A_{1}\right)
\end{array}\right.\right. \\
\text { и }\left\{\begin{array} { c } 
{ f _ { 0 } a _ { 1 } = b _ { 0 } a _ { 1 } } \\
{ f _ { 0 } c = b _ { 0 } c }
\end{array} \text { и } \left\{\begin{array}{c}
f_{1} a_{0}=b_{0} a_{0} \\
f_{1} a_{1}=b_{1} c
\end{array}\right.\right.
\end{gathered}
$$

и $\left(A_{4} ; b_{0}, b_{1}\right)$ является расслоенным произведением $\left(a_{1}, a_{0}\right)$ проекций $b_{0}, b_{1}$ относительно дискретов.

ОПРЕДЕЛЕНИЕ 2-5. Эскиз $\mathcal{E}$ есть база эскиза, для которой $\left(A_{3} ; a_{1}, a_{0}\right)$ является расслоенным произведением $\left(d_{0}, d_{1}\right)$ с проекциями $\left(a_{1}, a_{0}\right)$ относительно дискретов и

$$
e d_{0}=e d_{1}=I\left(A_{1}\right) \text { и с } d_{i}=a_{i} d_{i} \quad(i=0,1)
$$


и

$$
\Phi_{\mathcal{E}}\left(A_{4} ; b_{0}, b_{1}, e_{0}, e_{1}, f_{0}, f_{1}\right) \Rightarrow \bigwedge_{i=0,1} e_{i} c=I\left(A_{2}\right) \text { и } f_{0} c=f_{1} c
$$

Гомоморфизм $\mathcal{H}$ базисов эскизов из $\mathcal{E}$ в $\mathcal{E}^{\prime}$ задается тремя гомоморфизмами

$$
H_{i}: A_{i} \rightarrow A_{i}^{\prime}(i=1,2,3)
$$

Здесь

$$
\mathcal{H}=\left(H_{1}, H_{2}, H_{3}\right) \text { и } H_{i}: \mathcal{E} \rightarrow \mathcal{E}^{\prime} .
$$

Гомоморфизм $H(h)$ базисов эскизов, ассоииированных с функтором $h: A \rightarrow B$, определяется как:

$$
H(h)=\left(\left|h^{1}\right|,\left|h^{2}\right|,\left|h^{3}\right|\right) \text { или } H(h): \mathcal{E}(A) \rightarrow \mathcal{E}(B) .
$$

Гомоморфизм эскизов из эскиза $\mathcal{E}$ в $\mathcal{E}^{\prime}$ представляет собой гомоморфизм базисов эскизов $\left(H_{1}, H_{2}, H_{3}\right)$, для которых справедливо:

$$
e H_{2}=H_{1} e^{\prime} \text { и } H_{3} a_{0}^{\prime}=a_{0} H_{2} \text { и } H_{3} a_{1}^{\prime}=a_{1} H_{2} \text { и } H_{3} c^{\prime}=c H_{2} \text {. }
$$

ОПРЕДЕЛЕНИЕ композиции двух гомоморбизмов эскизов: пусть

$$
\mathcal{E} \stackrel{H}{\rightarrow} \mathcal{E}^{\prime} \stackrel{K}{\rightarrow} \mathcal{E}^{\prime \prime}
$$

где

$$
H=\left(H_{1}, H_{2}, H_{3}\right) \text { и } K=\left(K_{1}, K_{2}, K_{3}\right),
$$

тогда $H K: \mathcal{E} \rightarrow \mathcal{E}^{\prime \prime}$ есть

$$
H K=\left(H_{1} K_{1}, H_{2} K_{2}, H_{3} K_{3}\right) \text {. }
$$

Нетрудно убедиться, что в метакатегории эскизов объекты представляют собой эскизы, а стрелки будут гомоморфизмами эскизов.

Ax2-9 (конструкиия категорий и функторов эскизов). 
$1^{0} . \forall A \forall B \forall h: A \rightarrow B, \mathcal{E}(A)$ и $\mathcal{E}(B)$ являются эскизами и $H(h)$ есть гомоморфизм эскизов из $\mathcal{E}(A)$ в $\mathcal{E}(B)$.

$2^{0}$. Для любых эскизов $\mathcal{E}$ и $\mathcal{E}^{\prime}$, любых гомоморфизмов эскизов $H: \mathcal{E} \rightarrow \mathcal{E}^{\prime}$ существуют $A$ и $A^{\prime}$, и существует один и только один $h: A \rightarrow A^{\prime}$, и существует изоморфизм эскизов $\psi$ и $\psi^{\prime}$, такие, что диаграмма

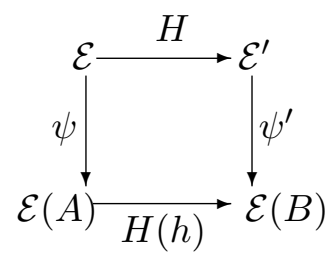

коммутативна.

\section{5 Неклассические элементарные теории категорий}

Существуют другие варианты аксиоматики теории категорий, позволяющие передать более сложные категорные структуры и конструкции. При этом они могут быть основаны на другом выборе нелогических предикатов. Так, в работе [9] строится паранепротиворечивая теория категорий $O B_{1}$, основанная на паранепротиворечивой логике да Косты, с одной стороны, и на теории категорий Убиньи-Боша $O B-$ с другой. В $O B_{1}$ в отличие от теорий, основанных на классической логике, мы можем конструировать и рассматривать противоречивые структуры, которые по своей природе не являются классическими теоретикомножественными структурами.

Действуя подобным образом, мы получаем паранепротиворечивую теорию категории категорий $P C C$, основанную на паранепротиворечивой логике и рассмотренной выше теории БланаДоннадью $C C$. Базисной логикой $P C C$ является $C_{1}^{=}$(паранепротиворечивая логика предикатов с равенством, надстроенная над логикой $C_{1}$ да Косты). Специфика аксиоматики $P C C$ сводится к следующему: если $A$ есть аксиома $C C$, то $A$ и $A^{*}\left(A^{*}\right.$ получается путем замены всех вхождений $\neg$ на $\neg$, где $\neg *$ $A$ означает $\neg A \wedge A^{o}$ и $A^{o}$ есть сокращение для $\left.\neg(A \wedge \neg A)\right)$ будут аксиомами $P C C$.

TEOPEMA 1. Пусть F будет формулой языка CC. Тогда если $F$ является теоремой $C C$, то $F^{*}$ есть теорема $P C C$. 
Доказательство. Является следствием аксиом $C C$ и $P C C$ и того факта, что $\neg$ * представляет собой классическое отрицание (см. [8]).

Q.E.D.

TEOPEMA 2. CC содержится в PCC.

Доказательство. Это следует из того факта, что $C_{1}$ лежит в основании $C_{1}^{=}$.

Q.E.D.

ТЕОРЕМА 3. РСС является паранепротиворечивой системой.

Доказательство. Если добавить расселово множество к $P C C$, т.е. множество $R=\{x: x \notin x\}$ где $x \notin x$ означает $\neg(x \in x)$ (слабое отрицание), то результирующая система не будет тривальной (см. [7]).

Таким образом, $P C C$ является очень сильной системой, в которой мы можем развить не только всю классическую математику, но также и исследовать такие «противоречивые» математические структуры, как расселово множество. В $P C C$ мы можем сконструировать другие типы «противоречивых» структур, не являющихся классическими теоретико-множественными структурами по своей природе. Например, мы можем рассмотреть некоторую форму парадокса Рассела. С этой целью введем следующее определение

$A \preccurlyeq B=_{\text {def }} \neg(A \rightarrow 1 \rightarrow|B|)$.

Рассмотрим категорию $K$, такую, что

$A \preccurlyeq K \leftrightarrow \neg(A \preccurlyeq A)$.

Как следствие, получаем

$K \preccurlyeq K \leftrightarrow \neg(K \preccurlyeq K)$

И

$K \preccurlyeq K \wedge \neg(K \preccurlyeq K)$.

Q.E.D.

Действуя сходным образом, мы можем рассмотреть квантовую теорию категории категорий $Q C C$, основанную на первопорядковой квантовой логике с равенством и рассмотренной выше теории Блана-Доннадью. Специфика языка квантовой логики, в частности принципиальное отсутствие в нем связки импликации, в этом случае, например, приводит к тому, что нам не требуется наличия в аксиоматике $Q C C$ аксиомы $\mathbf{A x 2 - 6}$, превращающей категорию категорий в декартово замкнутую категорию. 
Более того, наша категория категорий должна обладать структурой, подобной квантосу, в частности быть ортомодулярной категорией, чтобы получить возможность моделировать ортомодулярноое отрицание. Отсюда нам потребуются дополнительные аксиомы, позволяющие адекватно интерпретировать квантовую логику в категориях.

\section{Литература}

[1] Васюков В.Л.. Интерпретация релевантной логики в топосах // Логика и B.E.K. M., 2004. C. 112-121.

[2] Васюков В.Л. Квантовая логика. М.: Per Se, 2005.

[3] Васюков В.Л. Паранепротиворечивые категории для паранепротиворечивой логики // Логические исследования, вып. 17, М.-СПб.: Центр гуманитарных инициатив, 2011. С. 69-83.

[4] Голдблатm Р. Топосы. Категорный анализ логики. М., 1983.

[5] Blanc G., Donnadieu M. R. Axiomatisation de la categorie des categories // Cah. Topol. Geom. Different. XVII, 2, 1976. P. 1-38.

[6] Beall J.C. and Restall G. Logical Pluralism // Australasian Journal of Philosophy, 78, 2000. P. 475-493.

[7] N.C.A.da Costa. On Paraconsistent Set Theory // Logique et Analyse 115, 1986. P. $361-371$

[8] N.C.A.da Costa. Paraconsistent Mathematics // Frontiers of Paraconsistent Logic / D.Batens, C.Mortensen, G.Priest and J.-P. van Bendegem (eds.), Research Studies Press Ltd., Baldock, Hartfordshire, England, 2000. P. 166-179.

[9] N.C.A.da Costa, Bueno O., Volkov A. Outline of a Paraconsistent Category Theory // Alternative Logics. Do Science Need Them? / Weingartner P. (Ed.), Springer, Berlin, Heidelberg, New York, 2004. P. 95-114.

[10] Meyer R. K. Relevant Arithmetic // Bulletin of the Section of Logic of the Polish Academy of Sciences, 5, 1976. P. 133-137.

[11] Mortensen K. Inconsistent Mathematics. Dordrecht: Kluwer, 1995.

[12] Takeuti G. Quantum Set Theory // Current Issues on quantum logic / Beltrametti S., Fraassen B. Van (eds.). New York; London: Plenum, 1981. P. 303322.

[13] Takeuti G. and Titani S. Fuzzy Logic and Fuzzy Set Theory // Arch. Math. Log., 1992. P. 17-18.

[14] Vasyukov V. L. Paraconsistency in Categories // Frontiers of Paraconsistent Logic / D. Batens, C.Mortensen, G. Priest and J.-P. van Bendegem (eds.), Research Studies Press Ltd., Baldock, Hartfordshire, England, 2000. P. 263-278.

[15] Vasyukov V. L. Quantum Categories for Quantum Logic (в печати). 